\title{
Tests Based on Skewness and Kurtosis for Multivariate Normality
}

\author{
Namhyun $\operatorname{Kim}^{1, a}$ \\ ${ }^{a}$ Department of Science, Hongik University, Korea
}

\begin{abstract}
A measure of skewness and kurtosis is proposed to test multivariate normality. It is based on an empirical standardization using the scaled residuals of the observations. First, we consider the statistics that take the skewness or the kurtosis for each coordinate of the scaled residuals. The null distributions of the statistics converge very slowly to the asymptotic distributions; therefore, we apply a transformation of the skewness or the kurtosis to univariate normality for each coordinate. Size and power are investigated through simulation; consequently, the null distributions of the statistics from the transformed ones are quite well approximated to asymptotic distributions. A simulation study also shows that the combined statistics of skewness and kurtosis have moderate sensitivity of all alternatives under study, and they might be candidates for an omnibus test.
\end{abstract}

Keywords: Goodness of fit tests, multivariate normality, skewness, kurtosis, scaled residuals, empirical standardization, power comparison

\section{Introduction}

Classical multivariate analysis techniques require the assumption of multivariate normality; consequently, there are numerous test procedures to assess the assumption in the literature. For a general review, some references are Henze (2002), Henze and Zirkler (1990), Srivastava and Mudholkar (2003), and Thode (2002, Ch.9). For comparative studies in power, we refer to Farrell et al. (2007), Horswell and Looney (1992), Mecklin and Mundfrom (2005), and Romeu and Ozturk (1993).

Most multivariate techniques are often generalizations of univariate normality tests. $W$ test by Shapiro and Wilk (1965) and moment tests based on skewness and kurtosis are some of the most popular tests for univariate normality (Pearson et al., 1977). Shapiro and Wilk's $W$ test and the approximate tests to that (De Wet and Venter, 1972; Shapiro and Francia, 1972) have been generalized to multivariate cases by Fattorini (1986), Kim (2004a, 2005), Kim and Bickel (2003), Malkovich and Afifi (1973), Mudholkar et al. (1995), Royston (1983), Srivastava and Hui (1987), and Villasenor Alva and González Estrada (2009). As for skewness and kurtosis approaches, Mardia (1970, 1974)'s procedures are the most frequently used tests for multivariate normality. Kim (2004b), and Malkovich and Afifi (1973) generalized univariate moments to multivariate ones based on linear combinations of variates. Srivastava (1984) used principal components.

Numerous different testing procedures have been proposed in the literature to test multinormality; however, research has indicated no single test to be the most powerful for all situations. The Henze

\footnotetext{
This work was supported by 2015 Hongik University Research Fund. This work was done while the author was on sabbatical (Feb. 2015-Jan. 2016).

${ }^{1}$ Department of Science, Hongik University, 94 Wausan-Ro, Mapo-Gu, Seoul 121-791, Korea. E-mail: nhkim@hongik.ac.kr
} 
and Zirkler (1990) test is usually recommended as a formal goodness of fit test for multinormality since the invariance and consistency are proven theoretically and it has relatively good power across a wide range of alternatives (Farrell et al., 2007; Mecklin and Mundfrom, 2005). However some supplementary or less formal procedures (such as Mardia's skewness and kurtosis measures or some graphical procedures) should be followed up to help diagnose possible deviation from normality.

Mardia's procedures are among the most commonly used tests for multinormality; however, Baringhaus and Henze (1992), and Henze (1994) showed that Mardia's skewness and kurtosis are inconsistent against certain alternatives. Consequently, Mardia's procedures may have low power against some alternatives. It could also happen for other procedures for other procedures based on skewness or kurtosis. However, skewness and kurtosis can provide direct measure of departure from normality with strong points over other procedures.

In this paper, we propose multivariate skewness and kurtosis statistics based on an empirical standardization using the scaled residuals of the observations. Villasenor Alva and González Estrada (2009) used this idea to generalize Shapiro and Wilk's $W$ test that is closely related to Srivastava and Hui (1987)'s principal component approach. Section 2 presents the proposed test statistics for multivariate normality with the simulated critical values and $p$-values using asymptotic distributions. Section 3 contains a real data example and simulation study to compare the power performance of the statistics. Section 4 ends the paper with the concluding remarks.

\section{Test Statistics Based on Skewness and Kurtosis}

Let $\mathbf{X}_{1}, \mathbf{X}_{2}, \ldots, \mathbf{X}_{n}$ be $p$-dimensional independent and identically distributed (i.i.d.) random vectors, and let $N_{p}(\mu, \boldsymbol{\Sigma})$ be the $p$-variate multivariate normal distribution with mean vector $\mu$ and covariance matrix $\Sigma$. For the univariate normal distribution with mean $\mu$ and variance $\sigma^{2}$, we will use $N\left(\mu, \sigma^{2}\right)$ omitting the index.

We want to test the null hypothesis

$$
H_{0}: \mathbf{X}_{1}, \ldots, \mathbf{X}_{n} \text { is a sample from } N_{p}(\mu, \boldsymbol{\Sigma}) \text { for some } \mu \text { and } \boldsymbol{\Sigma} \text {. }
$$

First, we transform $\mathbf{X}_{1}, \mathbf{X}_{2}, \ldots, \mathbf{X}_{n}$ as

$$
\mathbf{Z}_{i}=\mathbf{S}^{*^{\prime}}\left(\mathbf{X}_{i}-\overline{\mathbf{X}}\right), \quad i=1, \ldots, n
$$

where ',' denotes a transpose, $\overline{\mathbf{X}}$ is the sample mean vector, $\overline{\mathbf{X}}=n^{-1} \sum_{j=1}^{n} \mathbf{X}_{j}, \mathbf{S}$ is the sample covariance matrix, $\mathbf{S}=n^{-1} \sum_{j=1}^{n}\left(\mathbf{X}_{j}-\overline{\mathbf{X}}\right)\left(\mathbf{X}_{j}-\overline{\mathbf{X}}\right)^{\prime}$, and $\mathbf{S}^{*}$ is defined by $\mathbf{S}^{*^{\prime}} \mathbf{S} \mathbf{S}^{*}=\mathbf{I}$. We call $\mathbf{Z}_{i}$ 's the scaled residuals, and it is well known $\mathbf{Z}_{1}, \ldots, \mathbf{Z}_{n}$ follow $N_{p}(\mathbf{0}, \mathbf{I})$ asymptotically if $\mathbf{X}_{1}, \mathbf{X}_{2}, \ldots, \mathbf{X}_{n}$ is a sample from $N_{p}(\mu, \boldsymbol{\Sigma})$, where $\mathbf{0}$ is the null vector of order $p$ and $\mathbf{I}$ is the identity matrix of order $p \times p$. The components of $\mathbf{Z}_{i}$, denoted by $\left(Z_{1 i}, Z_{2 i}, \ldots, Z_{p i}\right)$, are approximately independent standard normal $N(0,1)$ under the null hypothesis.

Therefore we can think of test statistics that consider the skewness or the kurtosis of each coordinate $\left(Z_{k 1}, Z_{k 2}, \ldots, Z_{k n}\right), k=1, \ldots, p$. As for skewness, it is defined by

$$
\sqrt{b_{1}(k)}=\frac{\sqrt{n} \sum_{j=1}^{n}\left(Z_{k j}-\bar{Z}_{k}\right)^{3}}{\left[\sum_{j=1}^{n}\left(Z_{k j}-\bar{Z}_{k}\right)^{2}\right]^{\frac{3}{2}}}=\frac{1}{n} \sum_{j=1}^{n} Z_{k j}^{3},
$$


and it is usually a two-sided test. Its square

$$
b_{1}(k)=\frac{n\left[\sum_{j=1}^{n}\left(Z_{k j}-\bar{Z}_{k}\right)^{3}\right]^{2}}{\left[\sum_{j=1}^{n}\left(Z_{k j}-\bar{Z}_{k}\right)^{2}\right]^{3}}=\left(\frac{1}{n} \sum_{j=1}^{n} Z_{k j}^{3}\right)^{2}
$$

is a one-sided test. The second equality in (2.2) or (2.3) follows from that each coordinate $\left(Z_{k 1}, Z_{k 2}\right.$, $\left.\ldots, Z_{k n}\right), k=1, \ldots, p$ has mean 0 and variance 1 .

To test the null hypothesis, we can think of the test statistic

$$
B_{1}=\max _{1 \leq k \leq p} b_{1}(k)
$$

since large value of $b_{1}(k)$ indicates a departure from normality. The statistic in (2.4) is closely related to Srivastava (1984)'s. Malkovich and Afifi (1973) generalized the univariate skewness and kurtosis to test multivariate normality using Roy's union-intersection principle (Roy, 1953), which is based on the fact that $\mathbf{c}^{\prime} \mathbf{X}$ follows a univariate normal for all $\mathbf{c}, \mathbf{c} \neq \mathbf{0}$, if $\mathbf{X}$ follows a multivariate normal. They investigated the skewness or the kurtosis of all the possible linear combinations that reduce to normal under the null hypothesis and tried to find a direction that gives furthest away from normal. Malkovich and Afifi (1973)'s multivariate skewness is as follows.

$$
b_{1, M}=\max _{\boldsymbol{c}, \boldsymbol{c} \neq \mathbf{0}} b_{1}(\boldsymbol{c})=\max _{\boldsymbol{c},\|\boldsymbol{c}\|=1} \frac{1}{n^{2}}\left[\sum_{j=1}^{n}\left(\boldsymbol{c}^{\prime} \boldsymbol{Z}_{j}\right)^{3}\right]^{2},
$$

where $\sqrt{b_{1}(\mathbf{c})}$ is the univariate skewness of $\boldsymbol{c}^{\prime} \boldsymbol{X}_{1}, \ldots, \boldsymbol{c}^{\prime} \boldsymbol{X}_{n}$ as in (2.2) by substituting $y_{j}=\boldsymbol{c}^{\prime} \boldsymbol{X}_{j}$ into $Z_{k j}, b_{1}(\mathbf{c})$ is its square likewise, and $Z_{j}$ is the scaled residual in (2.1). The second equality in (2.5) comes from the affine invariance with respect to addition of vectors and multiplication of nonsingular matrices (Kim, 2004b). However the statistic in (2.5) is hard to compute, especially when the dimension $p$ becomes large, practically when the dimension is $p>2$ (Horswell and Looney, 1992). Hence Kim (2004b) proposed an approximation to Malkovich and Afifi's statistic by selecting some directions that might achieve the maximum in (2.5) asymptotically. The statistic is included for comparison in Section 3. However, $B_{1}$ in (2.4) selects the vectors $\mathbf{c}$ as the unit vectors in each coordinate such that $\mathbf{c}=(1,0, \ldots, 0), \ldots,(0, \ldots, 0,1)$.

Under the null hypothesis of normality, $\sqrt{b_{1}(k)}$ is asymptotically normal with mean 0 and variance 6/n (Kendall and Stuart, 1977). Specifically,

$$
\sqrt{b_{1}(k)}\left[\frac{(n+1)(n+3)}{6(n-2)}\right]^{\frac{1}{2}} \stackrel{d}{\rightarrow} N(0,1) .
$$

Therefore the limit distribution of $B_{1}$ in (2.4) becomes

$$
B_{1}^{*}:=\frac{(n+1)(n+3)}{6(n-2)} B_{1} \stackrel{d}{\rightarrow} \max _{1 \leq k \leq p} V_{k}
$$

where $V_{1}, \ldots, V_{p}$ follow i.i.d. $\chi_{1}^{2}$, chi-square distribution with 1 degree of freedom, and we have

$$
\lim _{n \rightarrow \infty} P\left(B_{1}^{*} \leq x\right)=\left(P\left(V_{1} \leq x\right)\right)^{p} .
$$


However, the sample size must be somewhat large so that the normal approximation in (2.6) can be used. According to D'Agostino (1986), it appears to be valid for $n \geq 150$.

D'Agostino (1970) presented a transformation of the null distribution of $\sqrt{b_{1}(k)}$ to normality using a Johnson's unbounded $S_{U}$ curve. For each $\sqrt{b_{1}(k)}$, we compute

$$
\begin{aligned}
Y_{k}^{*} & =\sqrt{b_{1}(k)}\left[\frac{(n+1)(n+3)}{6(n-2)}\right]^{\frac{1}{2}}, \\
\beta_{2} & =\frac{3\left(n^{2}+27 n-70\right)(n+1)(n+3)}{(n-2)(n+5)(n+7)(n+9)}, \\
\omega^{2} & =\sqrt{2\left(\beta_{2}-1\right)}-1, \\
\delta & =\frac{1}{\sqrt{\log \omega}} \\
\alpha & =\sqrt{\frac{2}{\omega^{2}-1}}
\end{aligned}
$$

and

$$
T_{k}^{*}:=T_{k}^{*}\left(\sqrt{b_{1}(k)}\right)=\delta \log \left(\frac{Y_{k}^{*}}{\alpha}+\sqrt{\left(\frac{Y_{k}^{*}}{\alpha}\right)^{2}+1}\right) .
$$

The constant $\beta_{2}$ in the above transformation is based on the fourth standardized moment of the distribution of the skewness. The $T_{k}^{*}$ 's follow approximately a standard normal $N(0,1)$. It is applicable even for small sample sizes, $n \geq 8$. We propose the following statistics using $T_{k}^{*}$ in (2.8)

$$
S_{1}=\max _{1 \leq k \leq p}\left(T_{k}^{*}\right)^{2}, \quad S_{2}=\sum_{k=1}^{p}\left(T_{k}^{*}\right)^{2}
$$

as test statistics to test multivariate normality, then we have

$$
S_{1} \stackrel{d}{\rightarrow} \max _{1 \leq k \leq p} V_{k}, \quad \text { and } \quad S_{2} \stackrel{d}{\rightarrow} \chi_{p}^{2}
$$

Likewise $B_{1}$ in (2.4), large values of $S_{1}$ or $S_{2}$ will indicate non-normality.

For kurtosis, it is obtained by

$$
b_{2}(k)=\frac{n \sum_{j=1}^{n}\left(Z_{k j}-\bar{Z}_{k}\right)^{4}}{\left[\sum_{j=1}^{n}\left(Z_{k j}-\bar{Z}_{k}\right)^{2}\right]^{2}}=\frac{1}{n} \sum_{j=1}^{n} Z_{k j}^{4},
$$

and the kurtosis is asymptotically normal with mean 3 and variance $24 / n$ under normality. The following normal approximation is often used

$$
Y_{k}^{* *}:=\sqrt{\frac{(n+1)^{2}(n+3)(n+5)}{24 n(n-2)(n-3)}}\left(b_{2}(k)-\frac{3(n-1)}{(n+1)}\right) \stackrel{d}{\rightarrow} N(0,1)
$$


using the mean and variance of the kurtosis. A test statistic for multivariate normality could be

$$
B_{2}=\max _{1 \leq k \leq p}\left(b_{2}(k)-\frac{3(n-1)}{(n+1)}\right)^{2},
$$

and we have

$$
B_{2}^{* *}:=\frac{(n+1)^{2}(n+3)(n+5)}{24 n(n-2)(n-3)} B_{2} \stackrel{d}{\rightarrow} \max _{1 \leq k \leq p} V_{k} .
$$

The normal approximation for the kurtosis in (2.10) is slow and the sample size must be extremely large so that the approximation is valid. D'Agostino (1986) recommended it not be used because it should be over $n=1000$.

Likewise in the skewness, Malkovich and Afifi (1973)'s multivariate kurtosis is defined by

$$
b_{2, M}^{2}=\max _{\boldsymbol{c}, \boldsymbol{c} \neq \mathbf{0}}\left[b_{2}(\boldsymbol{c})-3\right]^{2}=\max _{\boldsymbol{c},\|\boldsymbol{c}\|=1}\left[\frac{1}{n} \sum_{j=1}^{n}\left(\boldsymbol{c}^{\prime} \boldsymbol{Z}_{j}\right)^{4}-3\right]^{2}
$$

with $b_{2}(\mathbf{c})$ the univariate kurtosis of $\boldsymbol{c}^{\prime} \boldsymbol{X}_{1}, \ldots, \boldsymbol{c}^{\prime} \boldsymbol{X}_{n}$ as in (2.9) plugging $y_{j}=\boldsymbol{c}^{\prime} \boldsymbol{X}_{j}$ into $Z_{k j}$. The approximate statistic is given in Kim (2004b).

Anscombe and Glynn (1983) showed a normal approximation for the kurtosis. Compute $\sqrt{\beta_{1}}$, the third standardized moment of kurtosis,

$$
\sqrt{\beta_{1}}=\frac{6\left(n^{2}-5 n+2\right)}{(n+7)(n+9)} \sqrt{\frac{6(n+3)(n+5)}{n(n-3)(n-2)}},
$$

and compute

$$
A=6+\frac{8}{\sqrt{\beta_{1}}}\left[\frac{2}{\sqrt{\beta_{1}}}+\sqrt{1+\frac{4}{\beta_{1}}}\right]
$$

Finally

$$
T_{k}^{* *}:=T_{k}^{* *}\left(b_{2}(k)\right)=\left[1-\frac{2}{9 A}-\left(\frac{1-(2 / A)}{1+Y_{k}^{* *} \sqrt{2 /(A-4)}}\right)^{\frac{1}{3}}\right] \frac{1}{\sqrt{2 /(9 A)}}
$$

follows approximately a standard normal $N(0,1)$. The $Y_{k}^{* *}$ is defined in $(2.10)$, and it is the standardized value of the kurtosis. The proposed statistic using $T_{k}^{* *}$ in (2.13) could be

$$
K_{1}=\max _{1 \leq k \leq p}\left(T_{k}^{* *}\right)^{2}, \quad K_{2}=\sum_{k=1}^{p}\left(T_{k}^{* *}\right)^{2}
$$

likewise in the skewness. They have

$$
K_{1} \stackrel{d}{\rightarrow} \max _{1 \leq k \leq p} V_{k}, \quad \text { and } \quad K_{2} \stackrel{d}{\rightarrow} \chi_{p}^{2}
$$


Table 1: Simulated critical values and $p$-values using asymptotic distribution for $p=2, \alpha=0.05$

\begin{tabular}{c|c|rrrrrrrrr}
\hline \hline Statistic & Values & $n=10$ & $n=20$ & $n=30$ & $n=40$ & $n=50$ & $n=100$ & $n=150$ & $n=200$ & $n=\infty$ \\
\hline$B_{1}$ & Critical value & 1.811 & 1.201 & 0.882 & 0.688 & 0.563 & 0.289 & 0.203 & 0.151 \\
\hline \multirow{2}{*}{$B_{1}^{*}$} & Critical value & 5.396 & 5.371 & 5.373 & 5.324 & 5.284 & 5.107 & 5.286 & 5.173 & 5.002 \\
& $p$-value & 0.040 & 0.041 & 0.040 & 0.042 & 0.043 & 0.047 & 0.043 & 0.045 & 0.050 \\
\hline \multirow{2}{*}{$S_{1}$} & Critical value & 5.317 & 5.057 & 5.010 & 4.855 & 5.044 & 5.007 & 4.978 & 5.095 & 5.002 \\
& $p$-value & 0.042 & 0.048 & 0.050 & 0.054 & 0.049 & 0.050 & 0.051 & 0.047 & 0.050 \\
\hline \multirow{2}{*}{$S_{2}$} & Critical value & 5.908 & 5.983 & 6.175 & 5.809 & 5.933 & 5.940 & 5.910 & 5.927 & 5.991 \\
& $p$-value & 0.052 & 0.050 & 0.046 & 0.055 & 0.051 & 0.051 & 0.052 & 0.052 & 0.050 \\
\hline \hline \multirow{2}{*}{$B_{2}$} & Critical value & 3.687 & 3.628 & 3.124 & 2.805 & 2.239 & 1.110 & 0.836 & 0.599 & \\
\hline \multirow{2}{*}{$B_{2}^{* *}$} & Critical value & 6.472 & 6.263 & 6.370 & 6.761 & 6.270 & 5.368 & 5.770 & 5.382 & 5.002 \\
& $p$-value & 0.022 & 0.025 & 0.023 & 0.019 & 0.024 & 0.041 & 0.032 & 0.040 & 0.050 \\
\hline \multirow{2}{*}{$K_{1}$} & Critical value & 4.479 & 5.027 & 4.913 & 4.917 & 5.219 & 5.059 & 5.080 & 5.238 & 5.002 \\
& $p$-value & 0.067 & 0.049 & 0.053 & 0.052 & 0.044 & 0.048 & 0.048 & 0.044 & 0.050 \\
\hline \multirow{2}{*}{$K_{2}$} & Critical value & 5.406 & 5.976 & 5.893 & 6.074 & 6.073 & 6.359 & 6.321 & 6.075 & 5.991 \\
& $p$-value & 0.067 & 0.050 & 0.053 & 0.048 & 0.048 & 0.042 & 0.042 & 0.048 & 0.050 \\
\hline \multirow{2}{*}{$C_{1}$} & Critical value & 5.284 & 5.658 & 5.997 & 6.069 & 6.261 & 6.317 & 6.352 & 6.171 & 6.205 \\
& $p$-value & 0.083 & 0.068 & 0.056 & 0.054 & 0.048 & 0.047 & 0.046 & 0.051 & 0.050 \\
\hline \multirow{2}{*}{$C_{2}$} & Critical value & 10.627 & 10.581 & 10.172 & 10.200 & 10.441 & 10.368 & 10.168 & 9.849 & 9.488 \\
& $p$-value & 0.031 & 0.032 & 0.038 & 0.037 & 0.034 & 0.035 & 0.038 & 0.043 & 0.050 \\
\hline \hline
\end{tabular}

Srivastava and Hui (1987) computed Shapiro and Wilk's $W$ statistics of the principal components, transformed them to normality, applied the transformation $-2 \ln \phi(\cdot)$ for them with $\phi$ the density of a standard normal and took a summation for asymptotic distribution $\chi_{2 p}^{2}$. When we apply $-2 \ln \phi(\cdot)$ to the transformed skewness $T_{k}^{*}$ or the transformed kurtosis $T_{k}^{* *}$ and make the statistic $-2 \sum_{k=1}^{p} \ln \phi\left(T_{k}^{*}\right)$ or $-2 \sum_{k=1}^{p} \ln \phi\left(T_{k}^{* *}\right)$, these statistics should not be used due to their extremely poor power.

Considerable attention has been paid to the omnibus tests that combine information from skewness and kurtosis. D'Agostino and Pearson (1973) proposed the statistic

$$
\left(T_{k}^{*}\left(\sqrt{b_{1}(k)}\right)\right)^{2}+\left(T_{k}^{* *}\left(b_{2}(k)\right)\right)^{2}
$$

when $k=1$ as an omnibus statistic for testing univariate normality and viewed it as $\chi_{2}^{2}$ although the two although two variables are not independent. D'Agostino and Pearson (1974), D'Agostino (1986), and Bowman and Shenton (1986) mentioned that they are uncorrelated and nearly independent, and $\chi_{2}^{2}$ approximation for the statistic is no problem for $n \geq 100$. Bowman and Shenton $(1975,1986)$ also gave the same format of the statistic; therefore, we may propose the following statistics

$$
C_{1}=\max _{1 \leq k \leq p}\left[\left(T_{k}^{*}\right)^{2},\left(T_{k}^{* *}\right)^{2}\right], \quad C_{2}=\sum_{k=1}^{p}\left(T_{k}^{*}\right)^{2}+\sum_{k=1}^{p}\left(T_{k}^{* *}\right)^{2}
$$

as $p$-dimensional omnibus test statistics, having the null distributions approximately $\max _{1 \leq k \leq 2 p} V_{k}$, $\chi_{2 p}^{2}$ respectively, with $V_{1}, \ldots, V_{2 p}$ i.i.d. $\chi_{1}^{2}$.

A simulation study was performed to study the null distributions of the proposed statistics. For each combination of dimensions $p=2,5$ and different sample sizes $n=10,20,30,40,50,100,150,200$, and for $p=10, n=30,40,50,100,150,200,250,300$, we generate $N=5,000$ random samples and calculate the statistics. Random samples are generated from independent $N_{p}(\mathbf{0}, \mathbf{I})$, since the distribution of the statistics do not depend on unknown parameters $\mu, \boldsymbol{\Sigma}$.

In Table 1-6, the simulated critical values and $p$-values for each statistic are given for $\alpha=0.05,0.10$. The simulated quantile values $k_{\alpha}$ are given in the tables. The $p$-values are obtained using the asymptotic distribution $\max _{1 \leq k \leq p} V_{k}$ for $B_{1}, B_{2}, S_{1}, K_{1}, \max _{1 \leq k \leq 2 p} V_{k}$ for $C_{1}, \chi_{p}^{2}$ for $S_{2}, K_{2}$, and $\chi_{2 p}^{2}$ for $C_{2}$. 
Table 2: Simulated critical values and $p$-values using asymptotic distribution for $p=2, \alpha=0.10$

\begin{tabular}{c|c|ccccccccc}
\hline \hline Statistic & Values & $n=10$ & $n=20$ & $n=30$ & $n=40$ & $n=50$ & $n=100$ & $n=150$ & $n=200$ & $n=\infty$ \\
\hline$B_{1}$ & Critical value & 1.334 & 0.867 & 0.625 & 0.493 & 0.418 & 0.216 & 0.151 & 0.115 \\
\hline \multirow{2}{*}{$B_{1}^{*}$} & Critical value & 3.975 & 3.876 & 3.808 & 3.813 & 3.923 & 3.824 & 3.919 & 3.938 & 3.798 \\
& $p$-value & 0.090 & 0.096 & 0.099 & 0.099 & 0.093 & 0.099 & 0.093 & 0.092 & 0.100 \\
\hline \multirow{2}{*}{$S_{1}$} & Critical value & 3.883 & 3.843 & 3.851 & 3.814 & 3.790 & 3.839 & 3.850 & 3.866 & 3.798 \\
& $p$-value & 0.095 & 0.097 & 0.097 & 0.099 & 0.100 & 0.098 & 0.097 & 0.096 & 0.100 \\
\hline \multirow{2}{*}{$S_{2}$} & Critical value & 4.521 & 4.529 & 4.645 & 4.572 & 4.613 & 4.562 & 4.691 & 4.537 & 4.605 \\
& $p$-value & 0.104 & 0.104 & 0.098 & 0.102 & 0.100 & 0.102 & 0.096 & 0.103 & 0.100 \\
\hline \hline \multirow{2}{*}{$B_{2}$} & Critical value & 2.150 & 1.957 & 1.736 & 1.556 & 1.224 & 0.712 & 0.507 & 0.402 & \\
\hline \multirow{2}{*}{$B_{2}^{* *}$} & Critical value & 3.775 & 3.378 & 3.54 & 3.749 & 3.429 & 3.443 & 3.498 & 3.608 & 3.798 \\
& $p$-value & 0.101 & 0.128 & 0.116 & 0.103 & 0.124 & 0.123 & 0.119 & 0.112 & 0.100 \\
\hline \multirow{2}{*}{$K_{1}$} & Critical value & 3.506 & 3.785 & 3.823 & 3.790 & 3.907 & 3.844 & 3.874 & 3.934 & 3.798 \\
& $p$-value & 0.119 & 0.101 & 0.099 & 0.100 & 0.094 & 0.097 & 0.096 & 0.092 & 0.100 \\
\hline \multirow{2}{*}{$K_{2}$} & Critical value & 4.228 & 4.562 & 4.511 & 4.713 & 4.615 & 4.727 & 4.825 & 4.602 & 4.605 \\
& $p$-value & 0.121 & 0.102 & 0.105 & 0.095 & 0.100 & 0.094 & 0.090 & 0.100 & 0.100 \\
\hline \multirow{2}{*}{$C_{1}$} & Critical value & 4.184 & 4.456 & 4.691 & 4.765 & 5.030 & 5.007 & 5.060 & 4.881 & 4.956 \\
& $p$-value & 0.154 & 0.132 & 0.116 & 0.111 & 0.096 & 0.097 & 0.094 & 0.104 & 0.100 \\
\hline \multirow{2}{*}{$C_{2}$} & Critical value & 7.934 & 7.886 & 7.936 & 7.797 & 8.290 & 8.073 & 8.092 & 7.817 & 7.779 \\
& $p$-value & 0.094 & 0.096 & 0.094 & 0.099 & 0.082 & 0.089 & 0.088 & 0.099 & 0.100 \\
\hline \hline
\end{tabular}

Table 3: Simulated critical values and $p$-values using asymptotic distribution for $p=5, \alpha=0.05$

\begin{tabular}{c|c|rrrrrrrrr}
\hline \hline Statistic & Values & $n=10$ & $n=20$ & $n=30$ & $n=40$ & $n=50$ & $n=100$ & $n=150$ & $n=200$ & $n=\infty$ \\
\hline$B_{1}$ & Critical value & 2.530 & 1.702 & 1.222 & 0.995 & 0.788 & 0.406 & 0.267 & 0.194 & \\
\hline \multirow{2}{*}{$B_{1}^{*}$} & Critical value & 7.538 & 7.614 & 7.442 & 7.691 & 7.397 & 7.180 & 6.936 & 6.677 & 6.599 \\
& $p$-value & 0.030 & 0.029 & 0.031 & 0.027 & 0.032 & 0.036 & 0.042 & 0.048 & 0.050 \\
\hline \multirow{2}{*}{$S_{1}$} & Critical value & 6.642 & 6.707 & 6.634 & 6.326 & 6.813 & 6.631 & 6.520 & 6.464 & 6.599 \\
& $p$-value & 0.049 & 0.047 & 0.049 & 0.058 & 0.044 & 0.049 & 0.052 & 0.054 & 0.050 \\
\hline \multirow{2}{*}{$S_{2}$} & Critical value & 11.278 & 10.910 & 11.255 & 11.245 & 11.065 & 11.141 & 11.228 & 11.118 & 11.070 \\
& $p$-value & 0.046 & 0.053 & 0.047 & 0.047 & 0.050 & 0.049 & 0.047 & 0.049 & 0.050 \\
\hline \hline$B_{2}$ & critical value & 6.545 & 6.805 & 5.514 & 4.642 & 4.025 & 2.198 & 1.306 & 0.970 & \\
\hline \multirow{2}{*}{$B_{2}^{* *}$} & Critical value & 11.491 & 11.748 & 11.244 & 11.187 & 11.273 & 10.628 & 9.019 & 8.713 & 6.599 \\
& $p$-value & 0.003 & 0.003 & 0.004 & 0.004 & 0.004 & 0.006 & 0.013 & 0.016 & 0.050 \\
\hline \multirow{2}{*}{$K_{1}$} & Critical value & 5.805 & 6.422 & 6.689 & 6.958 & 6.871 & 6.991 & 7.179 & 7.252 & 6.599 \\
& $p$-value & 0.077 & 0.055 & 0.048 & 0.041 & 0.043 & 0.040 & 0.036 & 0.035 & 0.050 \\
\hline \multirow{2}{*}{$K_{2}$} & Critical value & 10.033 & 10.552 & 11.096 & 11.483 & 11.343 & 11.550 & 11.616 & 11.600 & 11.070 \\
& $p$-value & 0.074 & 0.061 & 0.050 & 0.043 & 0.045 & 0.041 & 0.040 & 0.041 & 0.050 \\
\hline \hline \multirow{2}{*}{$C_{1}$} & Critical value & 6.783 & 7.279 & 7.495 & 7.710 & 7.830 & 8.063 & 8.134 & 8.236 & 7.838 \\
& $p$-value & 0.088 & 0.068 & 0.060 & 0.054 & 0.050 & 0.044 & 0.043 & 0.040 & 0.050 \\
\hline \multirow{2}{*}{$C_{2}$} & Critical value & 19.892 & 20.087 & 20.001 & 19.697 & 19.478 & 19.056 & 19.539 & 18.757 & 18.307 \\
& $p$-value & 0.030 & 0.028 & 0.029 & 0.032 & 0.035 & 0.040 & 0.034 & 0.043 & 0.050 \\
\hline \hline
\end{tabular}

They are $P\left(\max _{1 \leq k \leq p} V_{k} \geq k_{\alpha}\right), P\left(\max _{1 \leq k \leq 2 p} V_{k} \geq k_{\alpha}\right), P\left(\chi_{p}^{2} \geq k_{\alpha}\right)$, or $P\left(\chi_{2 p}^{2} \geq k_{\alpha}\right)$ for each case. As for $B_{1}, B_{2}$, the statistics $B_{1}^{*}$ in (2.7), $B_{2}^{* *}$ in (2.11) are the ones where suitable constants depending on $n$ are multiplied with corresponding asymptotic distributions. For skewness, the $p$-values are about right even for $B_{1}$ when $p=2$, however they become different from the given significance level $\alpha$ when $p=5,10$. Regarding $S_{1}, S_{2}$, the null distributions are well approximated by their asymptotic distributions $\max _{1 \leq k \leq p} V_{k}, \chi_{p}^{2}$, respectively even for small sample sizes. As for kurtosis, the $p$-values for $B_{2}$ show a considerable difference from the given level and are significantly worse for $p=5,10$. The null distribution of $B_{2}$ is extremely positively skewed, and asymptotic distribution should not be used. For $K_{1}, K_{2}$, and $C_{1}, C_{2}$, the sample size $n$ should be moderately large to provide the right significance level for $p=2,5$, and they are conservative for $p=10$. The asymptotic distributions 
Table 4: Simulated critical values and $p$-values using asymptotic distribution for $p=5, \alpha=0.10$

\begin{tabular}{c|c|rrrrrrrrr}
\hline \hline Statistic & Values & $n=10$ & $n=20$ & $n=30$ & $n=40$ & $n=50$ & $n=100$ & $n=150$ & $n=200$ & $n=\infty$ \\
\hline$B_{1}$ & Critical value & 1.956 & 1.336 & 0.948 & 0.760 & 0.612 & 0.313 & 0.215 & 0.160 \\
\hline \multirow{2}{*}{$B_{1}^{*}$} & Critical value & 5.828 & 5.974 & 5.773 & 5.874 & 5.744 & 5.540 & 5.593 & 5.499 & 5.339 \\
& $p$-value & 0.076 & 0.070 & 0.079 & 0.075 & 0.080 & 0.090 & 0.087 & 0.092 & 0.100 \\
\hline \multirow{2}{*}{$S_{1}$} & Critical value & 5.468 & 5.380 & 5.277 & 5.189 & 5.364 & 5.280 & 5.397 & 5.351 & 5.339 \\
& $p$-value & 0.093 & 0.098 & 0.103 & 0.109 & 0.099 & 0.103 & 0.097 & 0.099 & 0.100 \\
\hline \multirow{2}{*}{$S_{2}$} & Critical value & 9.447 & 9.261 & 9.425 & 9.407 & 9.181 & 9.301 & 9.389 & 9.295 & 9.236 \\
& $p$-value & 0.092 & 0.099 & 0.093 & 0.094 & 0.102 & 0.098 & 0.095 & 0.098 & 0.100 \\
\hline \hline \multirow{2}{*}{$B_{2}$} & Critical value & 4.210 & 4.340 & 3.293 & 2.984 & 2.639 & 1.333 & 0.867 & 0.671 & \\
\hline \multirow{2}{*}{$B_{2}^{* *}$} & Critical value & 7.391 & 7.492 & 6.714 & 7.191 & 7.392 & 6.446 & 5.984 & 6.029 & 5.339 \\
& $p$-value & 0.032 & 0.031 & 0.047 & 0.036 & 0.032 & 0.054 & 0.070 & 0.068 & 0.100 \\
\hline \multirow{2}{*}{$K_{1}$} & Critical value & 4.731 & 5.251 & 5.331 & 5.648 & 5.440 & 5.587 & 5.602 & 5.542 & 5.339 \\
& $p$-value & 0.140 & 0.105 & 0.100 & 0.084 & 0.095 & 0.087 & 0.087 & 0.089 & 0.100 \\
\hline \multirow{2}{*}{$K_{2}$} & Critical value & 8.525 & 8.767 & 9.255 & 9.444 & 9.337 & 9.635 & 9.576 & 9.575 & 9.236 \\
& $p$-value & 0.130 & 0.119 & 0.099 & 0.093 & 0.096 & 0.086 & 0.088 & 0.088 & 0.100 \\
\hline \multirow{2}{*}{$C_{1}$} & Critical value & 5.755 & 6.009 & 6.249 & 6.374 & 6.539 & 6.707 & 6.650 & 6.833 & 6.551 \\
& $p$-value & 0.153 & 0.134 & 0.118 & 0.110 & 0.101 & 0.092 & 0.095 & 0.086 & 0.100 \\
\hline \multirow{2}{*}{$C_{2}$} & Critical value & 16.604 & 16.944 & 16.863 & 16.731 & 16.854 & 16.285 & 16.541 & 16.244 & 15.987 \\
& $p$-value & 0.084 & 0.076 & 0.077 & 0.081 & 0.078 & 0.092 & 0.085 & 0.093 & 0.100 \\
\hline \hline
\end{tabular}

Table 5: Simulated critical values and $p$-values using asymptotic distribution for $p=10, \alpha=0.05$

\begin{tabular}{c|c|rrrrrrrrr}
\hline \hline Statistic & values & $n=30$ & $n=40$ & $n=50$ & $n=100$ & $n=150$ & $n=200$ & $n=250$ & $n=300$ & $n=\infty$ \\
\hline$B_{1}$ & Critical value & 1.523 & 1.177 & 0.964 & 0.492 & 0.326 & 0.246 & 0.192 & 0.160 \\
\multirow{2}{*}{$B_{1}^{*}$} & Critical value & 9.274 & 9.105 & 9.044 & 8.696 & 8.476 & 8.443 & 8.204 & 8.136 & 7.838 \\
& $p$-value & 0.023 & 0.025 & 0.026 & 0.031 & 0.035 & 0.036 & 0.041 & 0.043 & 0.050 \\
\hline \multirow{2}{*}{$S_{1}$} & Critical value & 7.823 & 7.826 & 7.732 & 7.968 & 8.056 & 7.814 & 7.726 & 7.720 & 7.838 \\
& $p$-value & 0.050 & 0.050 & 0.053 & 0.047 & 0.044 & 0.051 & 0.053 & 0.053 & 0.050 \\
\hline \multirow{2}{*}{$S_{2}$} & Critical value & 18.405 & 18.496 & 17.878 & 17.981 & 18.421 & 18.230 & 18.455 & 18.496 & 18.307 \\
& $p$-value & 0.049 & 0.047 & 0.057 & 0.055 & 0.048 & 0.051 & 0.048 & 0.047 & 0.050 \\
\hline \hline \multirow{2}{*}{$B_{2}$} & Critical value & 8.378 & 7.063 & 5.959 & 3.005 & 1.926 & 1.331 & 1.077 & 0.843 & \\
\hline \multirow{2}{*}{$B_{2}^{* *}$} & Critical value & 17.084 & 17.022 & 16.689 & 14.533 & 13.299 & 11.954 & 11.906 & 11.073 & 7.838 \\
& $p$-value & 0.000 & 0.000 & 0.000 & 0.001 & 0.003 & 0.005 & 0.006 & 0.009 & 0.050 \\
\hline \multirow{2}{*}{$K_{1}$} & Critical value & 7.974 & 8.269 & 8.527 & 8.777 & 8.676 & 8.702 & 8.472 & 8.557 & 7.838 \\
& $p$-value & 0.046 & 0.040 & 0.034 & 0.030 & 0.032 & 0.031 & 0.035 & 0.034 & 0.050 \\
\hline \multirow{2}{*}{$K_{2}$} & Critical value & 18.602 & 19.047 & 19.012 & 19.296 & 19.273 & 18.889 & 18.711 & 18.411 & 18.307 \\
& $p$-value & 0.046 & 0.040 & 0.040 & 0.037 & 0.037 & 0.042 & 0.044 & 0.048 & 0.050 \\
\hline \multirow{2}{*}{$C_{1}$} & Critical value & 8.885 & 9.200 & 9.488 & 9.522 & 9.409 & 9.178 & 9.618 & 9.351 & 9.906 \\
& $p$-value & 0.056 & 0.047 & 0.041 & 0.040 & 0.042 & 0.048 & 0.038 & 0.044 & 0.050 \\
\hline \multirow{2}{*}{$C_{2}$} & Critical value & 34.307 & 34.379 & 34.146 & 33.755 & 33.170 & 33.543 & 32.669 & 32.303 & 31.410 \\
& $p$-value & 0.024 & 0.024 & 0.025 & 0.028 & 0.032 & 0.029 & 0.037 & 0.040 & 0.050 \\
\hline \hline
\end{tabular}

approximate the null distributions fairly well for skewness statistics rather than kurtosis statistics or combined statistics. This can be explained by the slow normal approximation for one dimensional kurtosis in (2.10).

\section{Example and Simulation Results}

\subsection{Example}

We consider the data set of Rao (1948) that consists of the thickness of bark deposit on 28 cork trees measured by the weight of cork borings from the north $(\mathrm{N})$, east $(\mathrm{E})$, west $(\mathrm{W})$, and south $(\mathrm{S})$. He 
Table 6: Simulated critical values and $p$-values using asymptotic distribution for $p=10, \alpha=0.10$

\begin{tabular}{c|c|rrrrrrrrr}
\hline \hline Statistic & Values & \multicolumn{1}{|c}{$n=30$} & $n=40$ & $n=50$ & $n=100$ & $n=150$ & $n=200$ & $n=250$ & $n=300$ & $n=\infty$ \\
\hline$B_{1}$ & Critical value & 1.190 & 0.943 & 0.780 & 0.394 & 0.267 & 0.201 & 0.157 & 0.132 \\
\hline \multirow{2}{*}{$B_{1}^{*}$} & Critical value & 7.247 & 7.288 & 7.325 & 6.973 & 6.950 & 6.899 & 6.709 & 6.738 & 6.551 \\
& $p$-value & 0.069 & 0.067 & 0.066 & 0.080 & 0.081 & 0.083 & 0.092 & 0.090 & 0.100 \\
\hline \multirow{2}{*}{$S_{1}$} & Critical value & 6.455 & 6.620 & 6.524 & 6.687 & 6.732 & 6.593 & 6.508 & 6.538 & 6.551 \\
& $p$-value & 0.105 & 0.096 & 0.101 & 0.093 & 0.091 & 0.098 & 0.102 & 0.101 & 0.100 \\
\hline \multirow{2}{*}{$S_{2}$} & Critical value & 16.177 & 6.198 & 15.814 & 15.910 & 16.131 & 16.008 & 16.059 & 16.136 & 15.987 \\
& $p$-value & 0.095 & 0.094 & 0.105 & 0.102 & 0.096 & 0.099 & 0.098 & 0.096 & 0.100 \\
\hline \hline \multirow{2}{*}{$B_{2}$} & Critical value & 5.613 & 4.661 & 3.800 & 2.035 & 1.338 & 0.954 & 0.782 & 0.618 & 8.118 \\
\hline \multirow{2}{*}{$B_{2}^{* *}$} & Critical value & 11.445 & 11.232 & 10.642 & 9.841 & 9.239 & 8.568 & 8.647 & 6.551 \\
& $p$-value & 0.007 & 0.008 & 0.011 & 0.017 & 0.023 & 0.034 & 0.032 & 0.043 & 0.100 \\
\hline \multirow{2}{*}{$K_{1}$} & Critical value & 6.665 & 6.884 & 6.983 & 7.168 & 6.993 & 7.208 & 7.026 & 6.978 & 6.551 \\
& $p$-value & 0.094 & 0.084 & 0.079 & 0.072 & 0.079 & 0.070 & 0.078 & 0.080 & 0.100 \\
\hline \multirow{2}{*}{$K_{2}$} & Critical value & 15.955 & 16.316 & 16.494 & 16.726 & 16.623 & 16.346 & 16.475 & 16.067 & 15.987 \\
& $p$-value & 0.101 & 0.091 & 0.086 & 0.081 & 0.083 & 0.090 & 0.087 & 0.098 & 0.100 \\
\hline \multirow{2}{*}{$C_{1}$} & Critical value & 7.461 & 7.700 & 7.974 & 8.038 & 8.082 & 7.928 & 8.096 & 7.893 & 7.790 \\
& $p$-value & 0.119 & 0.105 & 0.091 & 0.088 & 0.086 & 0.093 & 0.085 & 0.095 & 0.100 \\
\hline \multirow{2}{*}{$C_{2}$} & Critical value & 29.932 & 30.656 & 30.067 & 29.796 & 29.440 & 29.495 & 29.267 & 29.117 & 28.412 \\
& $p$-value & 0.071 & 0.060 & 0.069 & 0.073 & 0.079 & 0.078 & 0.083 & 0.085 & 0.100 \\
\hline \hline
\end{tabular}

Table 7: Statistics and $p$-values for the contrasts of Rao's bark deposit data

\begin{tabular}{c|ccc|ccc|cc}
\hline \hline & $B_{1}$ & $S_{1}$ & $S_{2}$ & $B_{2}$ & $K_{1}$ & $K_{2}$ & $C_{1}$ & $C_{2}$ \\
\hline Statistic & 0.333 & 2.004 & 3.680 & 0.943 & 2.378 & 4.212 & 2.378 & 7.892 \\
$p$-value & 0.516 & 0.495 & 0.451 & 0.532 & 0.409 & 0.378 & 0.650 & 0.444 \\
\hline \hline
\end{tabular}

selected three constraints to investigate if the thickness of bark deposit varies in the four directions,

$$
Y_{1}=N-E-W+S, \quad Y_{2}=S-W, \quad Y_{3}=N-S
$$

by the reason he explained in the paper, and tested $E\left(Y_{i}\right)=0, i=1,2,3$. The assumption of multinormality should be valid to test the problem by applying some techniques like Hotelling's $T^{2}$ test. Pearson (1956) also examined the data set in Example 2 in his paper.

For the contrasts $\left(Y_{1}, Y_{2}, Y_{3}\right)$, the skewness is $(0.662,0.228,-0.038)$, and the kurtosis is $(3.812$, $3.935,1.641)$. After transforming to $\mathbf{Z}_{i}$ in (2.1), the skewness in (2.2), and the kurtosis in (2.9) are

$$
\left(\sqrt{b_{1}(1)}, \sqrt{b_{1}(2)}, \sqrt{b_{1}(3)}\right)=(-0.577,-0.476,-0.21),
$$

and

$$
\left(b_{2}(1), b_{2}(2), b_{2}(3)\right)=(3.764,2.679,1.981) .
$$

Applying the transformations in (2.8) and (2.13) yields

$$
\left(T_{1}^{*}, T_{2}^{*}, T_{3}^{*}\right)=(-1.416,-1.180,-0.533), \quad \text { and } \quad\left(T_{1}^{*} *, T_{2}^{*}, T_{3}^{*}\right)=(1.353,0.051,-1.542) .
$$

Table 7 provides the statistics in this paper and the $p$-values. The $p$-values are computed using $\chi^{2}$ distributions. From the result, the multinormality of the contrasts could not be rejected that confirms Rao's test for contrasts as valid. Mardia (1975), and Srivastava and Hui (1987) also considered the data set and have the same conclusion for the contrasts; however, they have different results for the original data. 
Table 8: Power comparison of $b_{1, M}^{*}, B_{1}, S_{1}, S_{2}, b_{2, M}^{2 *}, B_{2}, K_{1}, K_{2}$, and $C_{1}, C_{2}(\alpha=0.05, d=2, n=20)$

\begin{tabular}{|c|c|c|c|c|c|c|c|c|c|c|}
\hline \multirow{2}{*}{ Alternative } & \multicolumn{4}{|c|}{ Group I } & \multicolumn{4}{|c|}{ Group II } & \multicolumn{2}{|c|}{ Group III } \\
\hline & $b_{1, M}^{*}$ & $B_{1}$ & $S_{1}$ & $S_{2}$ & $b_{2, M}^{2 *}$ & $B_{2}$ & $K_{1}$ & $K_{2}$ & $C_{1}$ & $C_{2}$ \\
\hline$N(0,1)^{2}$ & 5 & 5 & 4 & 5 & 5 & 5 & 4 & 4 & 4 & 4 \\
\hline$C(0,1)^{2}$ & 92 & 91 & 89 & 93 & 96 & 96 & 94 & 95 & 95 & 96 \\
\hline $\operatorname{Logis}(0,1)^{2}$ & 16 & 12 & 14 & 15 & 15 & 18 & 10 & 10 & 14 & 15 \\
\hline$\left(t_{2}\right)^{2}$ & 64 & 61 & 64 & 63 & 71 & 69 & 63 & 65 & 68 & 68 \\
\hline$\left(t_{5}\right)^{2}$ & 28 & 25 & 24 & 27 & 27 & 31 & 22 & 22 & 23 & 25 \\
\hline$B(1,1)^{2}$ & 1 & 1 & 0 & 1 & 0 & 0 & 16 & 18 & 12 & 6 \\
\hline$B(2,2)^{2}$ & 2 & 1 & 2 & 1 & 1 & 1 & 6 & 7 & 6 & 2 \\
\hline$B(1,2)^{2}$ & 7 & 8 & 8 & 9 & 3 & 2 & 10 & 8 & 10 & 6 \\
\hline $\exp (1)^{2}$ & 71 & 69 & 70 & 71 & 55 & 48 & 41 & 47 & 64 & 64 \\
\hline $\mathrm{LN}(0, .5)^{2}$ & 53 & 50 & 51 & 57 & 41 & 37 & 36 & 32 & 45 & 50 \\
\hline$\Gamma(0.5,1)^{2}$ & 91 & 87 & 86 & 91 & 74 & 72 & 62 & 68 & 87 & 85 \\
\hline$\Gamma(5,1)^{2}$ & 24 & 22 & 23 & 23 & 17 & 15 & 13 & 14 & 21 & 23 \\
\hline$\left(\chi_{5}^{2}\right)^{2}$ & 39 & 39 & 38 & 43 & 29 & 25 & 23 & 20 & 35 & 37 \\
\hline$\left(\chi_{15}^{2}\right)^{2}$ & 17 & 18 & 18 & 20 & 15 & 13 & 10 & 12 & 14 & 16 \\
\hline$N(0,1) * t_{5}$ & 16 & 19 & 15 & 17 & 18 & 18 & 15 & 15 & 17 & 16 \\
\hline$N(0,1) * B(1,1)$ & 2 & 3 & 2 & 2 & 3 & 2 & 16 & 16 & 12 & 7 \\
\hline$N(0,1) * \exp (1)$ & 47 & 43 & 43 & 43 & 31 & 30 & 21 & 23 & 39 & 35 \\
\hline$N(0,1) * \chi_{5}^{2}$ & 24 & 33 & 34 & 29 & 19 & 18 & 14 & 16 & 29 & 26 \\
\hline $\operatorname{NMIX}_{2}(.5,2,0,0)$ & 3 & 3 & 2 & 3 & 3 & 4 & 10 & 11 & 8 & 4 \\
\hline $\operatorname{NMIX}_{2}(.5,4,0,0)$ & 3 & 4 & 4 & 4 & 2 & 3 & 75 & 70 & 68 & 50 \\
\hline $\operatorname{NMIX}_{2}(.5,2, .9,0)$ & 22 & 14 & 14 & 16 & 16 & 21 & 18 & 21 & 22 & 20 \\
\hline $\operatorname{NMIX}_{2}(.5, .5, .9,0)$ & 14 & 16 & 17 & 18 & 18 & 22 & 15 & 17 & 19 & 19 \\
\hline $\operatorname{NMIX}_{2}(.5, .5, .9,-.9)$ & 37 & 34 & 36 & 36 & 35 & 36 & 27 & 31 & 38 & 35 \\
\hline
\end{tabular}

\subsection{Simulation results}

A simulation was performed to study the power of the proposed tests based on the skewness and kurtosis of the scaled residuals. For dimensions and sample sizes, $d=2, n=20,50, d=5, n=20,50$, and $d=10, n=50,100$, the power of the statistics is presented in Tables 8-13 under the significance level $\alpha=0.05$. We generated 1,000 samples from each of various alternative distributions. The alternatives included in the study are distributions with independent marginals and mixtures of normal distributions. The following notations are used. $N(0,1), C(0,1), \operatorname{Logis}(0,1)$, and $\exp (1)$ stand for a standard normal, Cauchy, logistic, and exponential distribution; $\chi_{k}^{2}$ and $t_{k}$ are for the chi-square, $t$ distribution with $k$ degrees of freedom, respectively; $\Gamma(a, b)$ is for the gamma distribution with density $b^{-a} \Gamma(a)^{-1} x^{a-1} \exp (-x / b), x>0 ; B(a, b)$ is for the beta distribution with density $B(a, b)^{-1} x^{a-1}(1-$ $x)^{b-1}, 0<x<1 ; \operatorname{LN}(a, b)$ is for the lognormal distribution with density $(\sqrt{2 \pi} b x)^{-1} \exp (-(\log x-$ $\left.a)^{2} / 2 b^{2}\right), x>0$. Here $N(0,1), C(0,1)$, $\operatorname{Logis}(0,1), t_{k}, B(1,1)$, and $B(2,2)$ are symmetric distributions, and the others are skewed distributions. The product of $k$ independent copies of $F_{1}$ is denoted by $F_{1}^{k}$. The alternatives $F_{1}^{k}$ with symmetric marginals $F_{1}$ such as $C(0,1)$, $\operatorname{Logis}(0,1), t_{k}, B(1,1)$, and $B(2,2)$ are in the first part of each table. $F_{1} * F_{2}$ denotes the distribution having independent marginal distributions $F_{1}$ and $F_{2}$. $\operatorname{NMIX}_{2}\left(\kappa, \delta, \rho_{1}, \rho_{2}\right)$ is for the bivariate normal mixture

$$
\kappa N_{2}\left(\left(\begin{array}{l}
0 \\
0
\end{array}\right),\left(\begin{array}{cc}
1 & \rho_{1} \\
\rho_{1} & 1
\end{array}\right)\right)+(1-\kappa) N_{2}\left(\left(\begin{array}{l}
\delta \\
\delta
\end{array}\right),\left(\begin{array}{cc}
1 & \rho_{2} \\
\rho_{2} & 1
\end{array}\right)\right)
$$

which is a correlated distribution.

In the study, we put the power of Kim (2004b)'s statistics $b_{1, M}^{*}, b_{2, M}^{2 *}$ for comparison. As we mentioned in Section 2, they are approximate statistics to Malkovich and Afifi (1973)'s skewness in 
Table 9: Power comparison of $b_{1, M}^{*}, B_{1}, S_{1}, S_{2}, b_{2, M}^{2 *}, B_{2}, K_{1}, K_{2}$, and $C_{1}, C_{2}(\alpha=0.05, d=2, n=50)$

\begin{tabular}{|c|c|c|c|c|c|c|c|c|c|c|}
\hline \multirow{2}{*}{ Alternative } & \multicolumn{4}{|c|}{ Group I } & \multicolumn{4}{|c|}{ Group II } & \multicolumn{2}{|c|}{ Group III } \\
\hline & $b_{1, M}^{*}$ & $B_{1}$ & $S_{1}$ & $S_{2}$ & $b_{2, M}^{2 *}$ & $B_{2}$ & $K_{1}$ & $K_{2}$ & $C_{1}$ & $C_{2}$ \\
\hline$N(0,1)^{2}$ & 5 & 4 & 6 & 4 & 4 & 5 & 6 & 6 & 5 & 5 \\
\hline$C(0,1)^{2}$ & 99 & 98 & 98 & 99 & 100 & 100 & 100 & 100 & 100 & 100 \\
\hline $\operatorname{Logis}(0,1)^{2}$ & 22 & 20 & 20 & 20 & 33 & 26 & 20 & 23 & 22 & 27 \\
\hline$\left(t_{2}\right)^{2}$ & 87 & 85 & 86 & 86 & 98 & 95 & 93 & 95 & 92 & 94 \\
\hline$\left(t_{5}\right)^{2}$ & 39 & 38 & 36 & 40 & 56 & 48 & 41 & 44 & 45 & 49 \\
\hline$B(1,1)^{2}$ & 0 & 0 & 0 & 0 & 0 & 0 & 50 & $\mathbf{5 7}$ & 44 & 37 \\
\hline$B(2,2)^{2}$ & 0 & 0 & 0 & 1 & 0 & 0 & 23 & 27 & 18 & 10 \\
\hline$B(1,2)^{2}$ & 17 & 18 & 18 & 25 & 1 & 1 & 18 & 18 & 20 & 20 \\
\hline $\exp (1)^{2}$ & 100 & 96 & 97 & 97 & 86 & 80 & 72 & 73 & 93 & 96 \\
\hline $\mathrm{LN}(0, .5)^{2}$ & 95 & 89 & 89 & 90 & 73 & 70 & 60 & 63 & 84 & 84 \\
\hline$\Gamma(0.5,1)^{2}$ & 100 & 100 & 99 & 100 & 96 & 94 & 92 & 95 & 99 & 99 \\
\hline$\Gamma(5,1)^{2}$ & 60 & 53 & 54 & 60 & 29 & 28 & 24 & 23 & 44 & 48 \\
\hline$\left(\chi_{5}^{2}\right)^{2}$ & 85 & 81 & 76 & 82 & 53 & 49 & 39 & 43 & 72 & 75 \\
\hline$\left(\chi_{15}^{2}\right)^{2}$ & 43 & 41 & 40 & 45 & 25 & 22 & 15 & 17 & 33 & 36 \\
\hline$N(0,1) * t_{5}$ & 27 & 28 & 22 & 25 & 33 & 34 & 27 & 28 & 28 & 30 \\
\hline$N(0,1) * B(1,1)$ & 3 & 2 & 3 & 2 & 2 & 2 & 72 & 72 & 66 & 54 \\
\hline$N(0,1) * \exp (1)$ & 93 & 82 & 82 & 82 & 62 & 55 & 43 & 46 & 74 & 73 \\
\hline$N(0,1) * \chi_{5}^{2}$ & 61 & 75 & 75 & 73 & 32 & 35 & 30 & 30 & 65 & 59 \\
\hline NMIX $_{2}(.5,2,0,0)$ & 2 & 3 & 3 & 2 & 3 & 3 & 39 & 38 & 31 & 25 \\
\hline $\operatorname{NMIX}_{2}(.5,4,0,0)$ & 2 & 2 & 2 & 3 & 2 & 28 & 99 & 99 & 99 & 99 \\
\hline $\operatorname{NMIX}_{2}(.5,2, .9,0)$ & 51 & 19 & 19 & 22 & 33 & 45 & 44 & 52 & 40 & 42 \\
\hline $\operatorname{NMIX}_{2}(.5, .5, .9,0)$ & 26 & 24 & 23 & 26 & 35 & 41 & 28 & 33 & 32 & 34 \\
\hline $\operatorname{NMIX}_{2}(.5, .5, .9,-.9)$ & 53 & 53 & 51 & 54 & 66 & 60 & 45 & 56 & 54 & 64 \\
\hline
\end{tabular}

(2.5) and kurtosis in (2.12), respectively. The statistics $b_{1, M}^{*}, b_{2, M}^{2 *}$ are as follows,

$$
\begin{aligned}
& b_{1, M}^{*}=\max _{1 \leq l \leq n} \frac{1}{n^{2}} \frac{\left[\sum_{j=1}^{n}\left(\left(\boldsymbol{X}_{l}-\overline{\boldsymbol{X}}\right)^{\prime} \boldsymbol{S}^{-1}\left(\boldsymbol{X}_{j}-\overline{\boldsymbol{X}}\right)\right)^{3}\right]^{2}}{\left[\left(\boldsymbol{X}_{l}-\overline{\boldsymbol{X}}\right)^{\prime} \boldsymbol{S}^{-1}\left(\boldsymbol{X}_{l}-\overline{\boldsymbol{X}}\right)\right]^{3}}, \\
& b_{2, M}^{2 *}=\max _{1 \leq l \leq n}\left[\frac{1}{n} \frac{\sum_{j=1}^{n}\left(\left(\boldsymbol{X}_{l}-\overline{\boldsymbol{X}}\right)^{\prime} \boldsymbol{S}^{-1}\left(\boldsymbol{X}_{j}-\overline{\boldsymbol{X}}\right)\right)^{4}}{\left[\left(\boldsymbol{X}_{l}-\overline{\boldsymbol{X}}\right)^{\prime} \boldsymbol{S}^{-1}\left(\boldsymbol{X}_{l}-\overline{\boldsymbol{X}}\right)\right]^{2}}-3\right]^{2} .
\end{aligned}
$$

From now on, we refer to Group I for statistics based on skewness, $b_{1, M}^{*}, B_{1}, S_{1}$, and $S_{2}$, Group II for $b_{2, M}^{*}, B_{2}, K_{1}$, and $K_{2}$ on kurtosis, and Group III for $C_{1}$ and $C_{2}$ that are the combined statistics shown in Tables 8-13.

Each number in Tables 8-13 represents the empirical power of each test in percentage form rounded to the next integer. From the first line of each table, we can see that every statistic seems to have good control of type I error due to the use of critical values from the simulation rather than from asymptotic distributions. The best power for each alternative is written in bold, except when the numbers are all the same as 100 .

The power study in Tables 8-13 indicates the following. First, statistics in each group show similar power, except that $K_{1}, K_{2}$ are more powerful when alternatives are $\operatorname{NMIX}_{2}(.5,2,0,0), \mathrm{NMIX}_{2}(.5,4,0$, $0)$, or with shorter tailed marginals beta distributions such as $B(1,1)^{k}, B(2,2)^{k}, B(1,2)^{k}$, and $N(0,1)^{k-1}$ * $B(1,1), k=2,5,10$. This happens for every combination of $d$ and $n$ considered in the study, and appears predominantly when the sample size $n$ is big relative to the dimension $p$. Statistics that belong 
Table 10: Power comparison of $b_{1, M}^{*}, B_{1}, S_{1}, S_{2}, b_{2, M}^{2 *}, B_{2}, K_{1}, K_{2}$, and $C_{1}, C_{2}(\alpha=0.05, d=5, n=20)$

\begin{tabular}{|c|c|c|c|c|c|c|c|c|c|c|}
\hline \multirow{2}{*}{ Alternative } & \multicolumn{4}{|c|}{ Group I } & \multicolumn{4}{|c|}{ Group II } & \multicolumn{2}{|c|}{ Group III } \\
\hline & $b_{1, M}^{*}$ & $B_{1}$ & $S_{1}$ & $S_{2}$ & $b_{2, M}^{2 *}$ & $B_{2}$ & $K_{1}$ & $K_{2}$ & $C_{1}$ & $C_{2}$ \\
\hline$N(0,1)^{5}$ & 4 & 6 & 5 & 4 & 6 & 6 & 6 & 6 & 4 & 4 \\
\hline$C(0,1)^{5}$ & 98 & 97 & 98 & 99 & 100 & 100 & 99 & 100 & 99 & 99 \\
\hline $\operatorname{Logis}(0,1)^{5}$ & 12 & 12 & 13 & 14 & 16 & 14 & 12 & 11 & 11 & 14 \\
\hline$\left(t_{2}\right)^{5}$ & 78 & 75 & 76 & 81 & 81 & 78 & 76 & 77 & 78 & 85 \\
\hline$\left(t_{5}\right)^{5}$ & 28 & 26 & 24 & 25 & 30 & 28 & 23 & 25 & 26 & 28 \\
\hline$B(1,1)^{5}$ & 1 & 2 & 2 & 2 & 1 & 3 & 6 & 9 & 6 & 3 \\
\hline$B(2,2)^{5}$ & 1 & 2 & 3 & 3 & 2 & 2 & 6 & 6 & 4 & 2 \\
\hline$B(1,2)^{5}$ & 3 & 5 & 5 & 7 & 4 & 4 & 7 & 7 & 6 & 6 \\
\hline $\exp (1)^{5}$ & 59 & 61 & 59 & 69 & 57 & 49 & 42 & 45 & 55 & 64 \\
\hline $\mathrm{LN}(0, .5)^{5}$ & 44 & 44 & 48 & 56 & 44 & 42 & 36 & 38 & 44 & 49 \\
\hline$\Gamma(0.5,1)^{5}$ & 83 & 82 & 82 & 89 & 81 & 74 & 62 & 74 & 80 & 88 \\
\hline$\Gamma(5,1)^{5}$ & 15 & 18 & 20 & 21 & 19 & 15 & 11 & 13 & 17 & 16 \\
\hline$\left(\chi_{5}^{2}\right)^{5}$ & 30 & 32 & 31 & 35 & 28 & 27 & 23 & 20 & 29 & 35 \\
\hline$\left(\chi_{15}^{2}\right)^{5}$ & 11 & 13 & 13 & 18 & 14 & 12 & 8 & 10 & 12 & 14 \\
\hline$N(0,1)^{4} * t_{5}$ & 9 & 11 & 11 & 11 & 10 & 12 & 10 & 11 & 11 & 12 \\
\hline$N(0,1)^{4} * B(1,1)$ & 4 & 4 & 3 & 4 & 4 & 4 & 5 & 8 & 5 & 4 \\
\hline$N(0,1)^{4} * \exp (1)$ & 21 & 18 & 17 & 18 & 18 & 14 & 14 & 12 & 16 & 15 \\
\hline$N(0,1)^{4} * \chi_{5}^{2}$ & 11 & 24 & 22 & 22 & 11 & 17 & 11 & 14 & 19 & 17 \\
\hline
\end{tabular}

Table 11: Power comparison of $b_{1, M}^{*}, B_{1}, S_{1}, S_{2}, b_{2, M}^{2 *}, B_{2}, K_{1}, K_{2}$, and $C_{1}, C_{2}(\alpha=0.05, d=5, n=50)$

\begin{tabular}{|c|c|c|c|c|c|c|c|c|c|c|}
\hline \multirow{2}{*}{ Alternative } & \multicolumn{4}{|c|}{ Group I } & \multicolumn{4}{|c|}{ Group II } & \multicolumn{2}{|c|}{ Group III } \\
\hline & $b_{1, M}^{*}$ & $B_{1}$ & $S_{1}$ & $S_{2}$ & $b_{2, M}^{2 *}$ & $B_{2}$ & $K_{1}$ & $K_{2}$ & $C_{1}$ & $C_{2}$ \\
\hline$N(0,1)^{5}$ & 6 & 4 & 4 & 4 & 5 & 5 & 5 & 4 & 5 & 6 \\
\hline$C(0,1)^{5}$ & 100 & 100 & 100 & 100 & 100 & 100 & 100 & 100 & 100 & 100 \\
\hline $\operatorname{Logis}(0,1)^{5}$ & 30 & 18 & 18 & 20 & 30 & 26 & 17 & 18 & 18 & 24 \\
\hline$\left(t_{2}\right)^{5}$ & 98 & 95 & 94 & 96 & 100 & 99 & 98 & 100 & 98 & 99 \\
\hline$\left(t_{5}\right)^{5}$ & 51 & 40 & 42 & 46 & 60 & 55 & 43 & 46 & 43 & 54 \\
\hline$B(1,1)^{5}$ & 0 & 2 & 1 & 1 & 0 & 1 & 22 & 25 & 16 & 10 \\
\hline$B(2,2)^{5}$ & 1 & 1 & 1 & 1 & 0 & 1 & 11 & 12 & 8 & 4 \\
\hline$B(1,2)^{5}$ & 2 & 7 & 7 & 11 & 1 & 2 & 9 & 10 & 8 & 8 \\
\hline $\exp (1)^{5}$ & 99 & 94 & 91 & 97 & 91 & 82 & 73 & 81 & 91 & 96 \\
\hline $\mathrm{LN}(0, .5)^{5}$ & 94 & 87 & 86 & 91 & 82 & 74 & 64 & 70 & 79 & 89 \\
\hline$\Gamma(0.5,1)^{5}$ & 100 & 99 & 100 & 100 & 99 & 98 & 94 & 98 & 99 & 100 \\
\hline$\Gamma(5,1)^{5}$ & 50 & 41 & 39 & 49 & 34 & 28 & 22 & 20 & 36 & 44 \\
\hline$\left(\chi_{5}^{2}\right)^{5}$ & 80 & 67 & 67 & 75 & 57 & 46 & 36 & 40 & 64 & 69 \\
\hline$\left(\chi_{15}^{2}\right)^{5}$ & 39 & 29 & 30 & 36 & 27 & 17 & 15 & 16 & 24 & 32 \\
\hline$N(0,1)^{4} * t_{5}$ & 19 & 17 & 16 & 18 & 22 & 25 & 20 & 21 & 22 & 21 \\
\hline$N(0,1)^{4} * B(1,1)$ & 4 & 3 & 4 & 4 & 3 & 4 & 42 & 37 & 34 & 24 \\
\hline$N(0,1)^{4} * \exp (1)$ & 59 & 39 & 40 & 48 & 40 & 27 & 26 & 21 & 36 & 37 \\
\hline$N(0,1)^{4} * \chi_{5}^{2}$ & 32 & 61 & 62 & 58 & 19 & 26 & 25 & 22 & 55 & 47 \\
\hline
\end{tabular}

to Group I or Group II show very poor power against the above alternatives; however, $K_{1}, K_{2}$ in Group II and $C_{1}, C_{2}$ in Group III have relatively good power, and $K_{1}, K_{2}$ is slightly superior to $C_{1}, C_{2}$ against the alternatives.

Second, Group I statistics based on skewness seem to have better power than Group II statistics based on kurtosis against alternatives with skewed marginal distributions such as $\exp (1)^{k}, \mathrm{LN}(0, .5)^{k}$, $\Gamma(.5,1)^{k}, \Gamma(5,1)^{k},\left(\chi_{5}^{2}\right)^{k},\left(\chi_{15}^{2}\right)^{k}, N(0,1)^{k-1} * \exp (1)$, and $N(0,1)^{k-1} * \chi_{5}^{2}$. Group I have similar or better power to Group III combined statistics against these alternatives. 
Table 12: Power comparison of $B_{1}, S_{1}, S_{2}, B_{2}, K_{1}, K_{2}$, and $C_{1}, C_{2}(\alpha=0.05, d=10, n=50)$

\begin{tabular}{|c|c|c|c|c|c|c|c|c|}
\hline \multirow{2}{*}{ Alternative } & \multicolumn{3}{|c|}{ Group I } & \multicolumn{3}{|c|}{ Group II } & \multicolumn{2}{|c|}{ Group III } \\
\hline & $B_{1}$ & $S_{1}$ & $S_{2}$ & $B_{2}$ & $K_{1}$ & $K_{2}$ & $C_{1}$ & $C_{2}$ \\
\hline$N(0,1)^{10}$ & 4 & 6 & 5 & 4 & 4 & 4 & 5 & 5 \\
\hline$C(0,1)^{10}$ & 100 & 100 & 100 & 100 & 100 & 100 & 100 & 100 \\
\hline $\operatorname{Logis}(0,1)^{10}$ & 16 & 18 & 17 & 16 & 12 & 15 & 12 & 18 \\
\hline$\left(t_{2}\right)^{10}$ & 98 & 98 & 100 & 99 & 99 & 100 & 99 & 100 \\
\hline$\left(t_{5}\right)^{10}$ & 44 & 44 & 48 & 51 & 40 & 45 & 43 & 50 \\
\hline$B(1,1)^{10}$ & 2 & 2 & 2 & 2 & 7 & 10 & 6 & 4 \\
\hline$B(2,2)^{10}$ & 2 & 2 & 2 & 2 & 7 & 6 & 6 & 3 \\
\hline$B(1,2)^{10}$ & 4 & 5 & 7 & 3 & 5 & 4 & 7 & 5 \\
\hline $\exp (1)^{10}$ & 87 & 88 & 95 & 76 & 61 & 77 & 81 & 92 \\
\hline $\mathrm{LN}(0, .5)^{10}$ & 81 & 80 & 87 & 69 & 58 & 68 & 73 & 85 \\
\hline$\Gamma(0.5,1)^{10}$ & 98 & 98 & 100 & 96 & 90 & 97 & 97 & 100 \\
\hline$\Gamma(5,1)^{10}$ & 29 & 31 & 41 & 21 & 12 & 17 & 24 & 28 \\
\hline$\left(\chi_{5}^{2}\right)^{10}$ & 54 & 54 & 66 & 38 & 27 & 33 & 43 & 57 \\
\hline$\left(\chi_{15}^{2}\right)^{10}$ & 23 & 24 & 28 & 15 & 12 & 11 & 15 & 20 \\
\hline$N(0,1)^{9} * t_{5}$ & 14 & 15 & 15 & 17 & 14 & 14 & 14 & 15 \\
\hline$N(0,1)^{9} * B(1,1)$ & 4 & 4 & 5 & 4 & 13 & 12 & 11 & 8 \\
\hline$N(0,1)^{9} * \exp (1)$ & 22 & 23 & 19 & 16 & 12 & 12 & 16 & 18 \\
\hline$N(0,1)^{9} * \chi_{5}^{2}$ & 53 & 54 & 45 & 23 & 18 & 17 & 41 & 34 \\
\hline
\end{tabular}

Table 13: Power comparison of $B_{1}, S_{1}, S_{2}, B_{2}, K_{1}, K_{2}$, and $C_{1}, C_{2}(\alpha=0.05, d=10, n=100)$

\begin{tabular}{|c|c|c|c|c|c|c|c|c|}
\hline \multirow{2}{*}{ Alternative } & \multicolumn{3}{|c|}{ Group I } & \multicolumn{3}{|c|}{ Group II } & \multicolumn{2}{|c|}{ Group III } \\
\hline & $B_{1}$ & $S_{1}$ & $S_{2}$ & $B_{2}$ & $K_{1}$ & $K_{2}$ & $C_{1}$ & $C_{2}$ \\
\hline$N(0,1)^{10}$ & 5 & 4 & 5 & 4 & 5 & 5 & 6 & 5 \\
\hline$C(0,1)^{10}$ & 100 & 100 & 100 & 100 & 100 & 100 & 100 & 100 \\
\hline $\operatorname{Logis}(0,1)^{10}$ & 20 & 16 & 25 & 29 & 15 & 22 & 18 & 27 \\
\hline$\left(t_{2}\right)^{10}$ & 100 & 100 & 100 & 100 & 100 & 100 & 100 & 100 \\
\hline$\left(t_{5}\right)^{10}$ & 57 & 56 & 62 & 75 & 64 & 72 & 63 & 76 \\
\hline$B(1,1)^{10}$ & 1 & 1 & 1 & 1 & 19 & 21 & 14 & 7 \\
\hline$B(2,2)^{10}$ & 2 & 2 & 1 & 2 & 9 & 12 & 8 & 4 \\
\hline$B(1,2)^{10}$ & 8 & 7 & 12 & 2 & 8 & 8 & 9 & 10 \\
\hline $\exp (1)^{10}$ & 99 & 97 & 100 & 95 & 88 & 95 & 98 & 100 \\
\hline $\mathrm{LN}(0, .5)^{10}$ & 96 & 96 & 99 & 88 & 80 & 91 & 94 & 98 \\
\hline$\Gamma(0.5,1)^{10}$ & 100 & 100 & 100 & 100 & 100 & 100 & 100 & 100 \\
\hline$\Gamma(5,1)^{10}$ & 52 & 51 & 67 & 32 & 20 & 23 & 41 & 56 \\
\hline$\left(\chi_{5}^{2}\right)^{10}$ & 81 & 79 & 90 & 58 & 43 & 55 & 73 & 88 \\
\hline$\left(\chi_{15}^{2}\right)^{10}$ & 33 & 33 & 49 & 24 & 15 & 16 & 27 & 38 \\
\hline$N(0,1)^{9} * t_{5}$ & 21 & 22 & 20 & 29 & 27 & 23 & 26 & 25 \\
\hline$N(0,1)^{9} * B(1,1)$ & 6 & 4 & 3 & 4 & 67 & 56 & 65 & 41 \\
\hline$N(0,1)^{9} * \exp (1)$ & 33 & 31 & 34 & 21 & 18 & 17 & 28 & 30 \\
\hline$N(0,1)^{9} * \chi_{5}^{2}$ & 93 & 92 & 80 & 43 & 31 & 28 & 86 & 64 \\
\hline
\end{tabular}

Third, we see that the statistic $b_{2, M}^{2 *}$ is more sensitive than the other statistics in Group II, $B_{2}, K_{1}, K_{2}$, except the alternatives with beta marginals or some normal mixtures. This might be explained by the fact that $b_{2, M}^{2 *}$ is trying to search the direction of vector that gives at least normal to the univariate one. For alternatives with beta marginals, $K_{1}$ and $K_{2}$ show superior power.

In general, it seems Group III statistics $C_{1}, C_{2}$ have relatively good power for all alternatives under study. They are likely to have the merits of both Group I and Group II, and they might be considered omnibus tests, although they are not uniformly the most powerful, and the sample size needs to be moderately large to use asymptotic distribution. 


\section{Concluding Remarks}

In this study, we propose test statistics based on the skewness and kurtosis of the scaled residuals for testing multinormality. We consider the skewness or the kurtosis in each coordinate of the scaled residuals, and transform them to normality in each coordinate. The null distribution of the statistic can be approximated by simple distribution, and the approximation is adequate by applying the transformation to normality. Through the simulation study, the combined statistics of skewness and kurtosis show moderate sensitivity for all alternatives under study.

\section{References}

Anscombe, F. J. and Glynn, W. J. (1983). Distribution of the kurtosis statistic $b_{2}$ for normal samples, Biometrika, 70, 227-234.

Baringhaus, L. and Henze, N. (1992). Limit distributions for Mardia's measure of multivariate skewness, Annals of Statistics, 20, 1889-1902.

Bowman, K. O. and Shenton, L. R. (1975). Omnibus test contours for departures from normality based on $\sqrt{b_{1}}$ and $b_{2}$, Biometrika, 62, 243-250.

Bowman, K. O. and Shenton, L. R. (1986). Moment $\left(\sqrt{b_{1}}, b_{2}\right)$ Techniques, In R. B. D'Agostino and M. A. Stephens (Eds.), Goodness-of-Fit Techniques, Marcel Dekker, New York, 279-329.

D'Agostino, R. B. (1970). Transformation to normality of the null distribution of $g_{1}$, Biometrika, 57, 679-681.

D’Agostino, R. B. (1986). Tests for the normal distribution. In R. B. D’Agostino and M. A. Stephens (Eds.), Goodness-of-Fit Techniques, Marcel Dekker, New York, 367-420.

D'Agostino, R. B. and Pearson, E. S. (1973). Tests for departure from normality: Empirical results for the distributions of $b_{2}$ and $\sqrt{b_{1}}$, Biometrika, 60, 613-622.

D'Agostino, R. B. and Pearson, E. S. (1974). Correction and amendment: Tests for departure from normality: Empirical results for the distributions of $b_{2}$ and $\sqrt{b_{1}}$, Biometrika, 61, 647.

De Wet, T. and Venter, J. H. (1972). Asymptotic distributions of certain test criteria of normality, South African Statistical Journal, 6, 135-149.

Farrell, P. J., Salibian-Barrera, M. and Naczk, K. (2007). On tests for multivariate normality and associated simulation studies, Journal of Statistical Computation and Simulation, 77, 1065-1080.

Fattorini, L. (1986). Remarks on the use of the Shapiro-Wilk statistic for testing multivariate normality, Statistica, 46, 209-217.

Henze, N. (1994). On Mardia's kurtosis for multivariate normality, Communications in StatisticsTheory and Methods, 23, 1031-1045.

Henze, N. (2002). Invariant tests for multivariate normality: A critical review, Statistical Papers, 43, 467-506.

Henze, N. and Zirkler, B. (1990). A class of invariant consistent tests for multivariate normality, Communications in Statistics-Theory and Methods, 19, 3539-3617.

Horswell, R. L. and Looney, S. W. (1992). A comparison of tests for multivariate normality that are based on measures of multivariate skewness and kurtosis, Journal of Statistical Computation and Simulation, 42, 21-38.

Kendall, M. and Stuart, A. (1977). The Advanced Theory of Statistics, Vol. I, MacMillan Publishing Co., New York.

Kim, N. (2004a). An approximate Shapiro-Wilk statistic for testing multivariate normality, The Korean Journal of Applied Statistics, 17, 35-47.

Kim, N. (2004b). Remarks on the use of multivariate skewness and kurtosis for testing multivariate 
normality, The Korean Journal of Applied Statistics, 17, 507-518.

Kim, N. (2005). The limit distribution of an invariant test statistic for multivariate normality, The Korean Communications in Statistics, 12, 71-86.

Kim, N. and Bickel, P. J. (2003). The limit distribution of a test statistic for bivariate normality, Statistica Sinica, 13, 327-349.

Malkovich, J. F. and Afifi, A. A. (1973). On tests for multivariate normality, Journal of the American Statistical Association, 68, 176-179.

Mardia, K. V. (1970). Measures of multivariate skewness and kurtosis with applications, Biometrika, 57, 519-530.

Mardia, K. V. (1974). Applications of some measures of multivariate skewness and kurtosis for testing normality and robustness studies, Sankhyā: The Indian Journal of Statistics Series B, 36, 115128.

Mardia, K. V. (1975). Assessment of multinormality and the robustness of Hotelling's $T^{2}$ test, Applied Statistics, 24, 163-171.

Mecklin, C. J. and Mundfrom, D. J. (2005). A Monte Carlo comparison of the Type I and Type II error rates of tests of multivariate normality, Journal of Statistical Computation and Simulation, 75, 93-107.

Mudholkar, G. S., Srivastava, D. K. and Lin, C. T. (1995). Some $p$-variate adaptations of the ShapiroWilk test of normality, Communications of Statistics-Theory and Methods, 24, 953-985.

Pearson, E. S. (1956). Some aspects of the geometry of statistics, Journal of the Royal Statistical Society Series A (General), 119, 125-146.

Pearson, E. S., D’Agostino, R. B. and Bowman, K. O. (1977). Tests for departure from normality: Comparison of powers, Biometrika, 64, 231-246.

Rao, C. R. (1948). Test of significance in multivariate analysis, Biometrika, 35, 58-79.

Romeu, J. L. and Ozturk, A. (1993). A comparative study of goodness-of-fit tests for multivariate normality, Journal of Multivariate Analysis, 46, 309-334.

Roy, S. N. (1953). On a heuristic method of test construction and its use in multivariate analysis, Annals of Mathematical Statistics, 24, 220-238.

Royston, J. P. (1983). Some techniques for accessing multivariate normality based on the ShapiroWilk W, Applied Statistics, 32, 121-133.

Shapiro, S. S. and Francia, R. S. (1972). An approximate analysis of variance test for normality, Journal of the American Statistical Association, 67, 215-216.

Shapiro, S. S. and Wilk, M. B. (1965). An analysis of variance test for normality (complete samples), Biometrika, 52, 591-611.

Srivastava, D. K. and Mudholkar, G. S. (2003). Goodness of fit tests for univariate and multivariate normal models, Handbook of Statistics, 22, 869-906.

Srivastava, M. S. (1984). A measure of skewness and kurtosis and a graphical method for assessing multivariate normality, Statistics \& Probability Letters, 2, 263-267.

Srivastava, M. S. and Hui, T. K. (1987). On assessing multivariate normality based on Shapiro-Wilk $W$ statistic, Statistics \& Probability Letters, 5, 15-18.

Thode, H. C. (2002). Testing for Normality, Marcel Dekker, New York.

Villasenor Alva, J. A. and González Estrada, E. (2009). A generalization of Shapiro-Wilk's test for multivariate normality, Communications in Statistics-Theory and Methods, 38, 1870-1833. 